\title{
204. Studies on the Wetting Effect and the Surface Tension of Solids
}

\author{
The Weakening Effect on the Young's Modulus of \\ Quenched Carbon Steel with Its Application for \\ Determining the Surface Tension
}

By Mizuho SATO

(Comm. by M. Masima, M.J.A., Dec. 13, 1954)

In his interesting paper concerning the wetting effect on some brittle solids, Benedicks $\mathrm{ks}^{12}$ has determined the weakening effect on the bending tensile strength of a quenched carbon steel due to distilled water to be -21 per cent at room temperature. It is really interesting to see that the wetting effect can also be confirmed in a metal as well as in glass and similar brittle solids. ${ }^{12}$ ) Since the weakening effect on glass has been observed not only in its breaking strength ${ }^{122}$ but also in its Young's modulus, ${ }^{3)}$ it is required to investigate whether the weakening effect on steel can also be found within its elastic limit or not. For this purpose, the author has carried out an experiment for observing the change in Y oung's modulus of steel due to wetting by liquids. According to this experiment, the modulus was lowered by wetting of methanol, glycerine and distilled water, showing that the lowering with increasing surface tensions of wetting liquids is represented by a descending straight line, as seen in the weakening effect on the breaking strength of glass and similar brittle solids. ${ }^{4)}$ On this basis, the surface tension of steel has been determined as reported below. Experiment has been carried out at room temperature of $18 \sim 20^{\circ} \mathrm{C}$. Each test in dry and wet states has been repeated at least 10 12 times.

The experimental procedure is the same as described in the previous paper. ${ }^{3)}$ As the specimen, a saw to be used for cutting of metals was cut into a rectangular plate whose cross-section is $6.725 \times 0.637 \mathrm{~mm}$; the span used was $26 \mathrm{~mm}$. Analysis of this specimen gave its compositions to be 1.33 per cent carbon, 0.17 per cent silicon and 0.27 per cent manganese. For this rectangular specimen,

1) C. Benedicks och G. Ruben: Jernk. Ann., 129, 37 (1945). C. Benedicks and R. Härdén: Ark. Fys., 3, Nr. 22, 407 (1951).

2) C. Benedicks and G. Ruben: Ind. Dia. Rev., 9, 107 (1949). T. C. Baker and F. W. Preston: J. Appl. Phys., 17, 170, 179 (1946). J. L. Glathart and F. W. Preston: J. Appl. Phys., 17, 189 (1946).

3) M. Sato: Proc. Japan Acad., 30, 445 (1954).

4) C. Benedicks and R. Härdén: Ark. Fys., 3, Nr. 22, 407 (1951). 
if the load and the amount of bending are $P$ and $\epsilon$ respectively, there holds the relation

$$
\epsilon=\frac{P l^{3}}{4 E b d^{3}} \quad \text { or } \quad E=\begin{gathered}
P l^{3} \\
4 \in b d^{3}
\end{gathered},
$$

where $E$ is the Young's modulus, $l$ the span, while $b$ and $d$ are the width and the thickness of the specimen respectively. Keeping $\epsilon$ to be constant in dry and wet states, we have the relation in a simple form of

$$
\frac{\Delta E}{E}=\frac{\Delta P}{P},
$$

showing that the relative changes in $E$ and $P$ due to wetting are equal to each other. Thus, the change in the modulus can be computed from that in loads which give an equal amount of bendings in dry and wet states. As proved already, ${ }^{37}$ in our apparatus $P$ is expressed by the formula

$$
P=88 p,
$$

where $p$ is the pressure observed by the manometer. So, the change in the modulus due to wetting can be determined by the change in the manometer reading for giving a constant amount of bending in dry and wet states.

We have at first observed the height difference $h$ in the U-tube manometer to be $4.03 \mathrm{~mm}$ for the amount of bending $10 \times 10^{-2} \mathrm{~mm}$ in dry state, from which the pressure $p$ was calculated to be

$$
p=2 \rho g h=1.07 \times 10^{4} \mathrm{dyn} / \mathrm{cm}^{2},
$$

being assumed that the density of mercury, $\rho$, and the acceleration of gravity, $g$, are $13.6 \mathrm{~g} / \mathrm{cm}^{3}$ and $980 \mathrm{~cm} / \mathrm{sec}^{2}$ respectively. Since the unit of the factor 88 in the expression of $P$ is $\mathrm{cm}^{2}$, as proved in the previous paper, ${ }^{32}$ the load becomes

$$
P=9.45 \times 10^{5} \text { dyn, }
$$

by which the above bending is produced. Substituting the numerical values of $P, \in, l, b$ and $d$ in the formula of $E$, we get

$$
E=23.90 \times 10^{11} \mathrm{dyn} / \mathrm{cm}^{2} \text {, }
$$

which seems to be of right order of magnitude, in comparison with the values $20.6 \sim 20.8 \times 10^{3} \mathrm{~kg} / \mathrm{mm}^{2}$ observed by Benedicks for a carbon steel whose compositions are 1.50 per cent carbon, $0.1 \sim 0.2$ per cent silicon and $0.2 \sim 0.3$ per cent manganese.

Next, we have dipped this specimen in distilled water at $18^{\circ} \mathrm{C}$ for $15 \mathrm{~min}$ and then carried out the bending test in the presence of this water, giving the same amount of bending as that in the dry condition. In this case the pressure exerted by piston stroke

5) C. Benedicks: International Critical Table, II, 516 (1927). 
was observed to be $8.58 \times 10^{3} \mathrm{dyn} / \mathrm{cm}^{2}$, from which the amount of load can be computed as follows:

$$
P^{\prime}=7.55 \times 10^{5} \text { dyn. }
$$

Consequently, the modulus in the presence of distilled water becomes

$$
E^{\prime}=19.08 \times 10^{11} \mathrm{dyn} / \mathrm{cm}^{2} \text {, }
$$

which is $c a .20$ per cent smaller than that obtained in the dry condition, that is,

$$
\frac{\Delta E}{E}=\frac{E^{\prime}-E}{E}=-0.2018,
$$

which agrees fairly well with Benedicks' datum -0.21 for the change in bending tensile strength of a quenched carbon steel. ${ }^{122}$ It seems, therefore, that the weakening effect on the modulus of quenched carbon steel is also the same as that on its bending tensile strength, as seen in glass. ${ }^{3)}$

Similarly, the weakening effect due to methanol and glycerine was observed, comparing the loads in wet states with that in dry state. Since the load which is to be applied on the specimen for giving the bending is always computed from the height difference $h$ in mercury column of our U-tube manometer, it is necessary to make a list of $h$ to be a function of surrounding media, as shown in the following table.

\begin{tabular}{c|c|c|c|c}
\hline Medium & Air & Methanol & Glycerine & Distilled water \\
\hline $\begin{array}{c}\text { Surface tension } \\
\text { dyn/cm }\end{array}$ & 0 & 22.7 & 62.5 & 72.0 \\
\hline$h \mathrm{~mm}$ & 4.03 & 3.78 & 3.27 & 3.22
\end{tabular}

As seen from this table, the larger the surface tension of wetting liquids is, the smaller the height difference becomes, showing that the load for giving a constant amount of bending decreases with increasing surface tensions of wetting liquids. This means that the weakening effect on the modulus increases with increasing surface tension of wetting liquids, so long as their wettabilities with the specimen are all so good that the angles of contact between them seem to be vanished actually. In the following table our experimental data concerning the changes in modulus due to wetting by methanol, glycerine and distilled water are given, in which $E=23.90 \times 10^{11} \mathrm{dyn} / \mathrm{cm}^{2}$ is taken.

These changes are represented in the annexed figure where the modulus of the specimen and the surface tension of the wetting liquids are taken along the ordinate and the abscissa respectively. As seen from this figure, the modulus decreases linearly with 


\begin{tabular}{c|c|c|c}
\hline Medium & Methanol & Glycerine & Distilled water \\
\hline$E^{\prime} \times 10^{-11} \mathrm{dyn} / \mathrm{cm}$ & 22.41 & 19.40 & 19.08 \\
\hline$\Delta E \times 10^{-11} \mathrm{dyn} / \mathrm{cm}$ & 1.49 & 4.50 & 4.82 \\
\hline$-\frac{\Delta E}{E} \times 10^{2}$ & 6.24 & 18.82 & 20.18
\end{tabular}

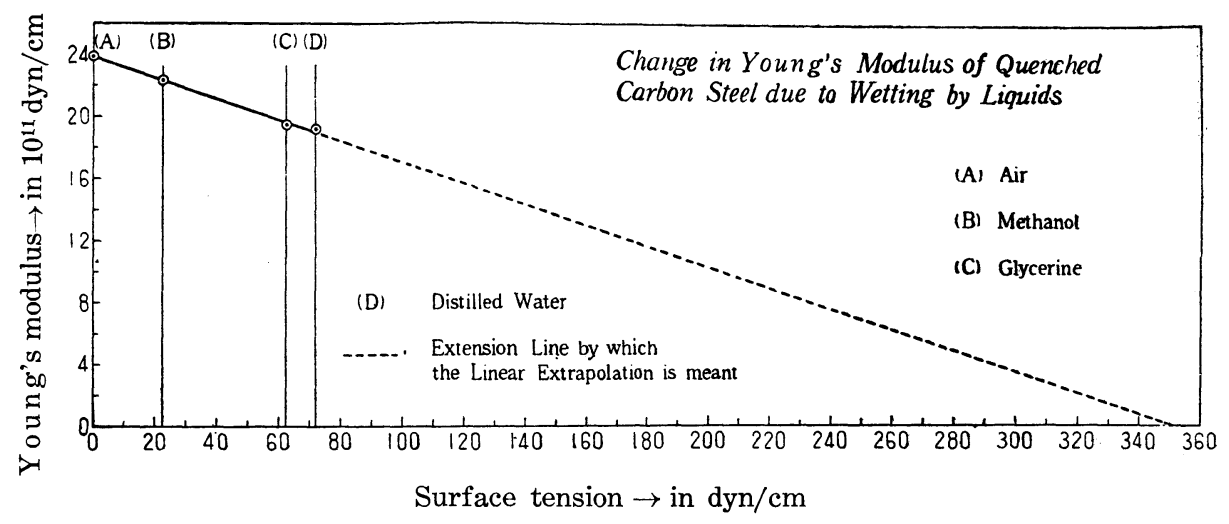

increasing surface tensions of wetting liquids, showing that the relation between them can be represented by a descending straight line as expected from the change in manometer reading.

This relation suggests how to determine the surface tension of the specimen. As treated in the previous paper, ${ }^{3)}$ this quantity $\gamma_{s}$ can be calculated by the formula

$$
\gamma_{s}=-\gamma_{\imath} \frac{\Delta E}{E}
$$

where $\gamma_{l}$ is the surface tension of wetting liquid. In the following table the results of calculation are given.

\begin{tabular}{|c|c|c|c|}
\hline Medium & Methanol & Glycerine & Distilled water \\
\hline$\gamma_{l} \mathrm{dyn} / \mathrm{cm}$ & 22.7 & 62.5 & 72.0 \\
\hline$-\frac{\Delta E}{E} \times 10^{2}$ & 6.24 & 18.82 & 20.18 \\
\hline$\gamma_{s} \mathrm{dyn} / \mathrm{cm}$ & 363.8 & 332.1 & 356.7 \\
\hline Mean of $\gamma_{s}$ & & $1 / \mathrm{cm}$ at & $0^{\circ} \mathrm{C}$ \\
\hline
\end{tabular}

As seen from this table, the surface tension of our quenched carbon steel can be determined to be $350 \sim 351 \mathrm{dyn} / \mathrm{cm}$ at $18 \sim 20^{\circ} \mathrm{C}$. 
According to $\mathrm{Benedicks},{ }^{1)}$ the weakening effect on the bending tensile strengh of his quenched carbon steel due to distilled water was -21 per cent as cited above. So, if this datum is taken for $\gamma_{\imath}=72.0 \mathrm{dyn} / \mathrm{cm}, \gamma_{s}$ becomes $343 \mathrm{dyn} / \mathrm{cm}$ which is close to our result $350 \sim 351 \mathrm{dyn} / \mathrm{cm}$ determined here.

The above calculation for determining the surface tension of specimen can be replaced by the graphical representation, extrapolating the linear relation between the lowering in the modulus and the surface tensions of wetting liquids, as shown by a dotted straight line in the annexed figure. The abscissa of the point of intersection of this line with the axis of surface tension is read to be $c a .350$ dyn/cm corresponding to the result of calculation. It is, however, inevitable that the result of calculation does not coincide exactly with that of graphical determination, because in the former the datum in dry state is assumed to be the standard by which the percentage change in the modulus due to wetting is computed, while in the latter all plots should be connected with a straight line by an interpolation supposing that the datum in the dry condition contains also an experimental error of the same order of magnitude as in the case of wet state. However that may be, it is interesting to see that the modulus of steel is lowered in the presence of some kind of surrounding medium, showing that this lowering can be applied for determining the surface tension of that steel. 